\title{
Características Antropométricas, Composición Corporal y Somatotipo de Jugadores de Tenis de Mesa de Alto Nivel
}

\author{
Anthropometric Characteristics, Body Composition and Somatotype \\ of High Level Table Tennis Players
}

\author{
Francisco Pradas de la Fuente*; José Antonio González Jurado*; \\ Edgardo Molina Sotomayor*** \& Carlos Castellar Otín*
}

PRADAS, F. ; GONZÁLEZ-JURADO, A.; MOLINA, E. \& CASTELLAR, C. Características antropométricas, composición corporal y somatotipo de jugadores de tenis de mesa de alto nivel. Int. J. Morphol., 31(4):1355-1364, 2013.

RESUMEN: El objetivo de este estudio fue describir las características antropométricas, la composición corporal y el somatotipo de una muestra internacional de jugadores de tenis de mesa de alto nivel clasificados entre los 150 mejores jugadores del mundo. En el estudio participaron un total de 31 jugadores (18 varones y 13 mujeres) de categoría absoluta. Todos los deportistas analizados en el estudio competían de manera regular en la Superdivisión, máxima categoría de la liga española y en pruebas internacionales de carácter oficial (ETTU e ITTF). La muestra fue distribuida en dos grupos atendiendo al sexo. Un total de 16 variables antropométricas fueron evaluadas. Se encontraron diferencias estadísticamente significativas en la masa, talla, IMC $(p<0,01)$, en cinco pliegues ( $p<0,05)$, en dos perímetros $(\mathrm{p}<0,01)$ y en todos los diámetros $(\mathrm{p} \leq 0,01)$. Asimismo se encontraron diferencias en el componente endomórfico y mesomórfico del somatotipo $(\mathrm{p}<0,02)$. El grupo masculino presentó una distribución del somatotipo mesomorfo balanceado $(2,5-4,6-2,6)$ mientras que el grupo femenino fue endo-mesomórfico $(4,0-3,4-2,9)$. Los resultados obtenidos sugieren un biotipo diferente a los datos de referencia, presentando los hombres menor endomorfia mientras que las mujeres alcanzan mayores niveles en su endomorfia y un descenso en la mesomorfia. Este estudio aporta datos biotipológicos actualizados de referencia para la población de elite de tenis de mesa.

PALABRAS CLAVE: Tenis de mesa; Elite; Antropometría; Composición corporal; Somatotipo.

\section{INTRODUCCIÓN}

El tenis de mesa es un deporte olímpico que se desarrolla sobre una superficie de juego rectangular de reducidas dimensiones: 2,74 m de largo por 1,525 $\mathrm{m}$ de ancho. $\mathrm{Su}$ juego se caracteriza por la ejecución de manera coordinada de diferentes acciones técnicas a máxima velocidad, con desplazamientos rápidos y cortos que implican continuos cambios de dirección. El desarrollo de este tipo de actividad supone a nivel físico un esfuerzo muscular muy severo como consecuencia de la explosividad que se requiere para desplazarse velozmente, golpear la pelota y recuperar la posición de base para preparar la siguiente acción técnica (Pradas et al., 2005).

Estudios realizados como los de Tang et al. (2013) ponen de manifiesto que en partidos de alta competición la pelota puede alcanzar velocidades próximas a los 126 kilómetros por hora. Ante esta situación deportiva el jugador se encuentra con la necesidad de tener que realizar en décimas de segundo y de manera simultánea un tratamiento de la información visual, un análisis de la misma y una respuesta técnica con la mayor precisión posible (Ripoll, 1989). Teniendo en cuenta estos factores, los requerimientos físicos necesarios en este deporte se orientan fundamentalmente al desarrollo de la fuerza activa, en su manifestación rápida, y la fuerza reactiva en su manifestación elástico-explosiva (Pradas et al., 2012a). La velocidad de desplazamiento, la velocidad gestual y el tiempo de reacción representan las manifestaciones elementales e integrales de la velocidad necesaria en este deporte, ya que se dan situaciones complejas que requieren un elevado nivel de coordinación, con reacciones de elección ante un objeto en movimiento que se desplaza a alta velocidad (Pradas et al., 2005).

Si analizamos a nivel fisiológico los estímulos que

\footnotetext{
* Facultad de Ciencias Humanas y de la Educación, Universidad de Zaragoza, España.

** Facultad del Deporte. Universidad Pablo de Olavide, España.

**** Facultad de Artes y Educación Física. Universidad Metropolitana de Ciencias de la Educación de Santiago de Chile, Chile.
} 
caracterizan esta disciplina deportiva podemos comprobar que las situaciones de juego que se desarrollan son de tipo intermitente, aeróbica-anaeróbica alterna, con un 40$50 \%$ para el metabolismo aeróbico, un $10-20 \%$ para el anaeróbico láctico y el resto para el anaeróbico aláctico (Faccini et al., 1989), con consumos medios de oxígeno durante los partidos de aproximadamente un $70 \%$ del máximo (Shepard \& Astrand, 1996) y frecuencias cardiacas de 145-160 latidos por minuto (Püschel, 1978; Allen, 1991).

Son numerosos los estudios realizados en deportes como el squash (Mellor et al., 1995; Alvero et al., 2006), el bádminton (Centeno et al., 1999; De Hoyo et al., 2007) o el tenis (Juzwiak et al., 2008; Sanchis et al., 1998; Sánchez-Muñoz et al., 2007; Solanellas et al., 1996; Torres-Luque et al., 2006), en los que se indaga y se analiza con precisión el biotipo de referencia en estas disciplinas deportivas. Sin embargo, son escasas las investigaciones que describen con exactitud las características antropométricas, la composición corporal y el somatotipo en el tenis de mesa de elite.

En la literatura científica podemos encontrar algunos estudios que describen las características antropométricas de jóvenes jugadores (Pradas et al., 2005; Pradas et al., 2007) o de deportistas de alto nivel (Biener \& Oechsli, 1979; Allen). Aunque en la actualidad estas investigaciones nos pueden resultar de gran interés son anteriores a la profunda transformación realizada en los últimos años por la Federación Internacional de Tenis de Mesa (ITTF) sobre el reglamento técnico de juego de este deporte. Dichas modificaciones han supuesto la creación de nuevas condiciones de juego que han afectado de manera importante la estructura y desarrollo del tenis de mesa, marcando un antes y un después en esta disciplina deportiva.

Considerando todo lo anterior, analizar el perfil antropométrico que caracteriza a los jugadores de tenis de mesa cobra una mayor relevancia. El objetivo de este estudio es el de describir, comparar y establecer el perfil antropométrico de jugadores de tenis de mesa de alto nivel de ambos géneros.

\section{MATERIAL Y MÉTODO}

Una muestra internacional de 31 jugadores de tenis de mesa de elite, 18 hombres (edad: $25,33 \pm 4,3$ años) y 13 mujeres (edad: $22,7 \pm 3,54$ años) participaron de manera voluntaria en este estudio. Los deportistas seleccionados tenían una experiencia media de $14,5 \pm 4$ años y entrena- ban un mínimo de 10 horas por semana en mesa, compitiendo de manera regular durante los últimos tres años en la liga de Superdivisión (máxima categoría española) y en pruebas internacionales de la European Table Tennis Union (ETTU) o de la ITTF. Los jugadores analizados se encontraban clasificados en el momento de realización del estudio entre los 150 mejores jugadores del mundo según el ranking oficial de la ITTF.

Para conocer las posibles diferencias existentes entre sexos la muestra fue dividida en dos grupos: hombres y mujeres. Las medidas realizadas para las determinaciones antropométricas incluyeron: masa corporal $(\mathrm{kg})$, altura $(\mathrm{cm})$, ocho pliegues (bíceps, tríceps, subescapular, suprailíaco, supraespinal, abdominal, muslo y pierna $(\mathrm{mm})$ ), cuatro perímetros (brazo relajado, brazo contraído y flexionado, muslo y pierna $(\mathrm{cm})$ ) y tres diámetros (bicondíleo femoral, biepicondíleo de húmero y biestiloideo radioulnar $(\mathrm{cm})$. Para el registro de todas las medidas se utilizaron los protocolos propuestos por la International Society for the Advancement of Anthropometry (ISAK) y las recomendaciones del Grupo Español de Cineantropometría (GREC) para determinar el perfil antropométrico. Todas las mediciones fueron realizadas por un experto acreditado por la ISAK ayudado por un asistente para registrar los valores obtenidos. Para asegurar una mayor validez y fiabilidad durante el proceso se siguieron las recomendaciones de Ross \& MarfellJones (1991) por las cuales es válido un error técnico de medida interevaluador e intraevaluador inferior a un $5 \%$ para los pliegues cutáneos y menor al 2\% para el resto de mediciones.

Para la medición de los pliegues se utilizó un plicómetro (Holtain Ltd, Crymych, UK) con una precision de $0,2 \mathrm{~mm}$. La altura y la masa corporal fueron medidas con una báscula modelo Seca 714 (Seca Instruments Ltd, Hamburg, Germany). Los diámetros fueron evaluados con un paquímetro y los perímetros con una cinta metálica flexible con precisión de $1 \mathrm{~mm}$ (Holtain Ltd, Crymych, UK) utilizándose un lápiz dermográfico.

A partir de las evaluaciones realizadas se calcularon diferentes índices y porcentajes con el fin de determinar el índice de masa corporal (IMC), la composición corporal y el somatotipo de los deportistas participantes en el estudio. El IMC fue calculado mediante la fórmula: [masa $(\mathrm{kg}) \mathrm{x}$ altura $(\mathrm{m})]^{2}$. La composición corporal fue estimada usando el método de De Rose \& Guimaraes (1980), basado en un modelo tetracompartimental de Matiegka determinándose los componentes graso, óseo, muscular y residual. El porcentaje de grasa corporal fue calculado utilizando la fórmula de Faulkner (1968), para el peso óseo se 
utilizó la de Von Doblen a partir de la modificación propuesta por Rocha (1975) y para el peso residual la de Würch (1974). El análisis del somatotipo se realizó utilizando el método propuesto por Heath-Carter (Carter \& Heath, 1990) obteniéndose el valor de los tres componentes: endomórfico, mesomórfico y ectomórfico.

Antes de su participación en el estudio todos los deportistas fueron informados de manera verbal del procedimiento a seguir en esta investigación, tras lo cual dieron su consentimiento por escrito. Esta investigación fue aprobada por el Comité Ético de Investigación Clínica del Gobierno de Aragón (España).

Se calcularon de forma separada para cada grupo la media y desviación estándar(DE). Los datos fueron analizados usando el paquete estadístico SPSS versión 20.0 (Inc, Chicago, Illinois). Para comprobar las posibles diferencias entre grupos se realizaron diferentes contrastes de hipótesis, comprobando la normalidad de la muestra mediante la prueba Shapiro-Wilk y una estimación de la homocedasticidad de varianzas a través de la prueba de Levene. Cuando la comparación de medias cumplía con las condiciones de normalidad se utilizó la prueba $\mathrm{T}$ de Student para datos independientes, aplicando la prueba no paramétrica U de Mann-Whitney en aquellas variables que no cumplían con los criterios de normalidad. Para todas las comparaciones se estableció un intervalo de confianza de $95 \%(\mathrm{p} \leq 0,05)$.

\section{RESULTADOS}

Las características generales de los deportistas participantes en el estudio se presentan diferenciadas por sexo en la Tabla I. Los datos se muestran como valores medios, rango (mínimo y máximo) y desviación típica. Los jugadores hombres presentan valores significativamente más elevados que las mujeres en las variables biométricas masa corporal ( $\mathrm{p}<0,001)$, talla $(\mathrm{p}<0,001)$ e IMC $(\mathrm{p} \leq 0,006)$.

Las variables asociadas al volumen de entrenamiento de la muestra se presentan en la Tabla II. Los jugadores señalaron que su frecuencia de entrenamiento y competición está por encima de las 180 sesiones al año, con periodos de entrenamientos que tienen una duración media de $10,8 \pm 1,06$ meses, distribuidos en 5,5 $\pm 0,7$ días a la semana y con una duración diaria media de 2,5 $\pm 0,6$ horas de entrenamiento en mesa en ambos géneros. Además, indicaron que dedican también un promedio de 2,5 $\pm 0,7$ horas semanales a la realización de un trabajo complementario de acondicionamiento físico fuera de la mesa, orientado hacia el desarrollo de la fuerza y de la capacidad aeróbica.

El perfil de los pliegues cutáneos, perímetros musculares y diámetros óseos se muestra en la Tabla III. El análisis efectuado sobre estas variables puso de manifiesto valores significativamente superiores $(\mathrm{p}<0,05)$ en el sexo femenino frente al masculino en todos los casos excepto para el pliegue

Tabla I. Comparación de características generales según sexo

\begin{tabular}{llcccc}
\hline \multicolumn{2}{c}{ Varones $(\mathbf{n = 1 8})$} & \multicolumn{3}{c}{ Mujeres $(\mathbf{n = 1 3})$} \\
\hline Variable & Media (DE) & Rango & Media (DE) & Rango & Valor p \\
Edad (años) & $25,3( \pm 4,3)$ & $19-38$ & $22,7( \pm 3,5)$ & $20-31$ & $\mathrm{~ns}$ \\
Masa (kg) & $71,9( \pm 9,1)$ & $56,3-89,6$ & $55,8( \pm 5,1)$ & $49-64,1$ & $<0,001^{\ddagger}$ \\
Talla $(\mathbf{c m})$ & $177( \pm 0,06)$ & $168-187$ & $165( \pm 0,06)$ & $159-175$ & $<0,001$ \\
IMC $\left(\mathbf{k g} / \mathbf{m}^{2}\right)$ & $22,9( \pm 2,2)$ & $19,95-27,25$ & $20,59( \pm 1,4)$ & $18,33-22,5$ & $\leq 0,006^{\ddagger}$ \\
\hline
\end{tabular}

(Prueba t de Student; \$Prueba U de Mann-Whitney; ns: no significativo).

Tabla II. Características del volumen de entrenamiento de la muestra.

\begin{tabular}{lccc}
\hline \multicolumn{4}{c}{ Entrenamientos } \\
Media (DE) & Mínimo & Máximo \\
\hline Experiencia en tenis de mesa (años) & $14,55( \pm 4,07)$ & 9 & 28 \\
Sesiones en la mesa (meses/año) & $10,8( \pm 1,06)$ & 10 & 12 \\
Sesiones en la mesa (días/semana) & $5,5( \pm 0,7)$ & 5 & 7 \\
Sesiones en la mesa (horas/sesión) & $2,5( \pm 0,6)$ & 2 & 5 \\
Sesiones en la mesa (horas/semana) & $20,5( \pm 4,2)$ & 10 & 22 \\
Sesiones complementarias (horas/semana) & $2,5( \pm 0,7)$ & 1 & 5 \\
\hline
\end{tabular}


Tabla III. Comparación de variables antropométricas según sexo.

\begin{tabular}{lccccc}
\hline & \multicolumn{2}{c}{ Varones $(\mathbf{n = 1 8})$} & \multicolumn{3}{c}{ Mujeres $(\mathbf{n = 1 3})$} \\
\hline Pliegues (mm) & Media (DE) & Rango & Media (DE) & Rango & Valor p \\
Tricipital & $8,4( \pm 2,9)$ & $4,8-14,4$ & $16,4( \pm 3)$ & $9,9-20,5$ & $<0,001 \neq$ \\
Bicipital & $3,8( \pm 1)$ & $2-5,8$ & $5,8( \pm 1,3)$ & $4,2-7,4$ & $<0,001$ \\
Subescapular & $9,6( \pm 2,7)$ & $6,4-17,2$ & $10,9( \pm 1,9)$ & $7,4-13,8$ & $\mathrm{~ns}$ \\
Suprailíaco & $12,1( \pm 5)$ & $5,8-22,9$ & $17,8( \pm 5,5)$ & $9,8-26,5$ & $\leq 0,005$ \\
Supraespinal & $8,6( \pm 3,8)$ & $4,6-19,2$ & $10,7( \pm 2,4)$ & $6,6-16,5$ & $<0,03^{\ddagger}$ \\
Abdominal & $13,8( \pm 6,8)$ & $5,6-26,4$ & $18,5( \pm 3,9)$ & $11,8-26,4$ & $<0,05^{\ddagger}$ \\
Muslo & $10,8( \pm 3,1)$ & $4,6-18$ & $24,6( \pm 3,5)$ & $18,8-29,3$ & $<0,001$ \\
Pierna & $7,1( \pm 2,7)$ & $4-12,4$ & $14,7( \pm 3,9)$ & $8,2-19,8$ & $<0,001 \ddagger$ \\
E6 pliegues & $58,6( \pm 18,3)$ & $31,6-95,2$ & $96( \pm 15,8)$ & $70,9-125,1$ & $<0,001$ \\
Perímetros (cm) & & & & & \\
Brazo flexionado & $31,6( \pm 2,5)$ & $28,5-37,7$ & $27,2( \pm 1,6)$ & $23,5-29,9$ & $<0,001$ \\
Muslo medio & $50,9( \pm 4,1)$ & $44-56,8$ & $48,7( \pm 3,4)$ & $44,3-54,2$ & $\mathrm{~ns}$ \\
Pierna & $36,2( \pm 2,7)$ & $31,8-41,6$ & $33,8( \pm 2,9)$ & $27,7-37,2$ & $<0,03$ \\
Diámetros (cm) & & & & & \\
Biepicondileo & $9,7( \pm 0,6)$ & $8,2-11$ & $8,9( \pm 0,5)$ & $7,8-9,9$ & $\leq 0,002$ \\
Biepicondiliano & $6,8( \pm 0,3)$ & $6,3-7,7$ & $5,6( \pm 0,5)$ & $4,6-6,4$ & $<0,001$ \\
Biestiloideo & $5,7( \pm 0,3)$ & $5,2-6,4$ & $4,9( \pm 0,3)$ & $4,1-5,4$ & $<0,001$ \\
\hline
\end{tabular}

(Prueba t de Student; $¥$ Prueba U de Mann-Whitney; ns: no significativo).

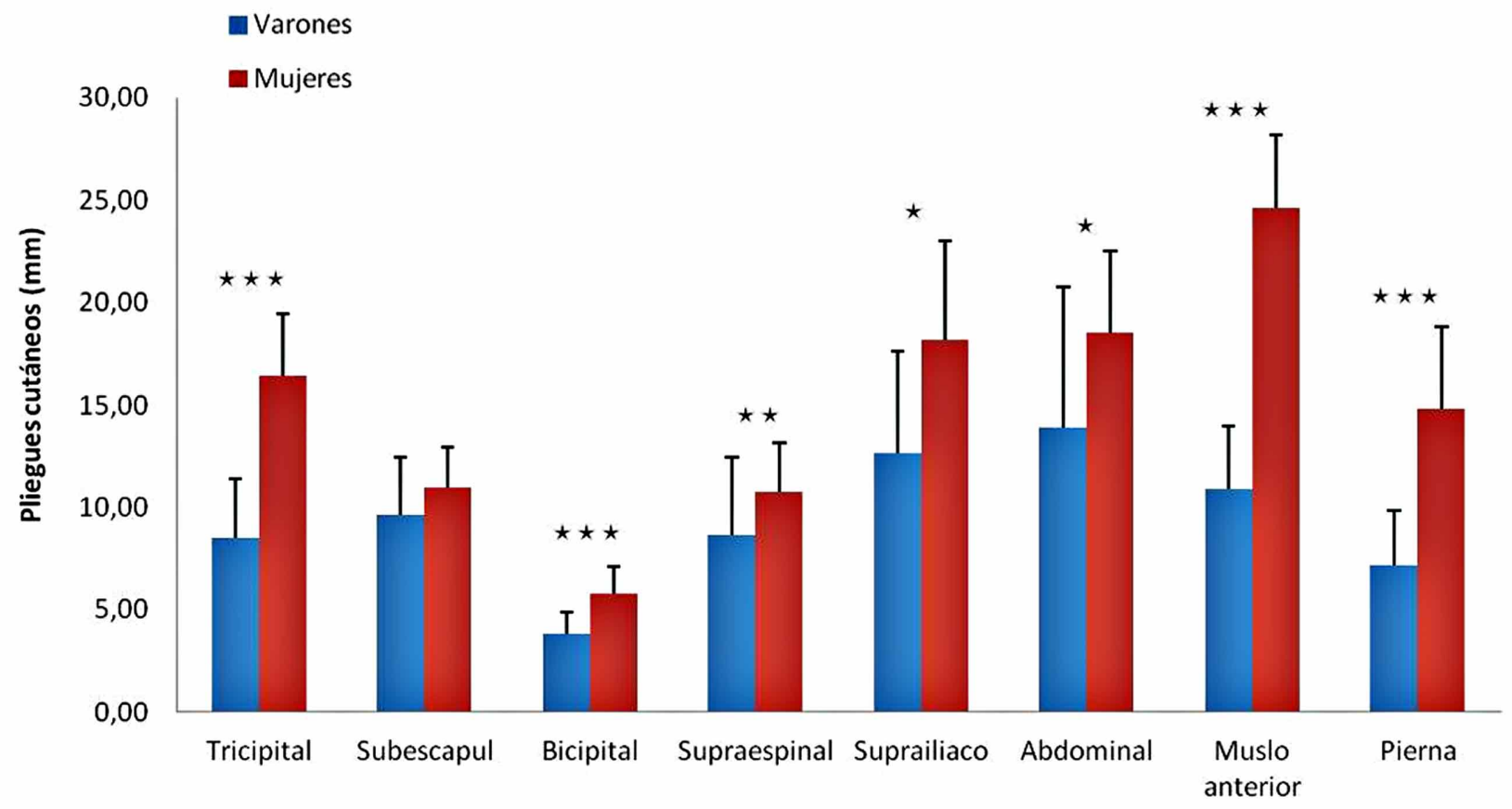

Fig. 1. Comparación de pliegues cutáneos de los jugadores de tenis de mesa según sexo. *p<0,05. **p<0,01. ***p<0,001.

subescapular y el perímetro del muslo. El sumatorio de los 8 pliegues analizados (Fig. 1) es de $75,1 \mathrm{~mm}$ y de $120 \mathrm{~mm}$ en hombres y mujeres respectivamente. En el sexo masculino el componente graso se distribuyó en un $60 \%$ para el tronco
$(44,7 \mathrm{~mm})$ y un $40 \%$ para los miembros $(30,4 \mathrm{~mm})$, mientras que en el sexo femenino esta relación se invierte siendo inferior para el tronco con un $49 \%(58,4 \mathrm{~mm})$ y superior para los miembros con un $51 \%(61,6 \mathrm{~mm})$. 
Usando las categorías propuestas por Carter \& Heath en la Tabla IV se presentan los datos obtenidos en la composición corporal. Los porcentajes de peso graso son menores $(\mathrm{p}<0,001)$ y los de peso muscular mayores $(\mathrm{p}<0,005)$ en los jugadores siendo estas diferencias estadísticamente significativas. Los jugadores de tenis de mesa de sexo masculino se caracterizan por poseer una mesomorfia dominante $(4,6 \pm 1,2)$ y una ectomorfia $(2,6 \pm 1,0)$ mayor que la endomorfia $(2,5 \pm 0,8)$ mientras que las mujeres presentaban una endomorfia dominante $(4,0 \pm 0,6)$ y una mesomorfia $(3,4 \pm 1,2)$ mayor que la ectomorfia $(2,9 \pm 0,9)$. Se ha encontrado significación estadística en los componentes endomórficos $(\mathrm{p}<0,001)$ y mesomórficos $(\mathrm{p}<0,02)$ del somatotipo manteniéndose similar en ambos sexos el ectomórfico.

Al comparar los individuos de sexo masculino con el femenino se encontraron diferencias estadísticamente significativas en el porcentaje de peso graso $(\mathrm{p}<0,001)$ y muscular $(\mathrm{p}<0,005)$, con un aumento notable en el porcentaje graso del $12 \%$ al $14,7 \%$. El porcentaje muscular se mantiene en ambos sexos en unos valores por encima del $45 \%$ siendo ligeramente superior en varones $(46,9 \%)$ frente a las mujeres $(45,7 \%)$, mientras que el porcentaje residual se mantiene constante en los dos grupos. En la Figura 2 se presenta un histograma con los porcentajes de los diferentes compartimentos corporales (graso, muscular, óseo y residual).

En las Figuras 3 y 4 se muestra la representación gráfica del somatotipo o somatocarta en donde aparecen reflejados todos los valores individuales y el promedio de la muestra analizada. El índice de dispersión del somatotipo (SDI) calculado para valorar la homogeneidad de los grupos estudiados indica que en los jugadores de ambos sexos existía una heterogeneidad, siendo el resultado del SDI en hombres de 3,84 y en mujeres de 3,43. Se considera que existen diferencias estadísticamente significativas cuando SDI es $\geq 2(p<0,05)$. La distancia de dispersión del somatotipo (DDS) fue de 1,01 y 1,94 en hombres y mujeres respectivamente. En las mujeres se encuentra muy próximo a obtener diferencias estadísticamente significativas (DDS $\geq 2$ ). En el cálculo de la distancia morfogénica del somatotipo (SAD) se obtuvieron valores de 0,64 y 0,8 en hombres y mujeres respectivamente. A mayores valores del SAD mayor será la diferencia entre los somatotipos.

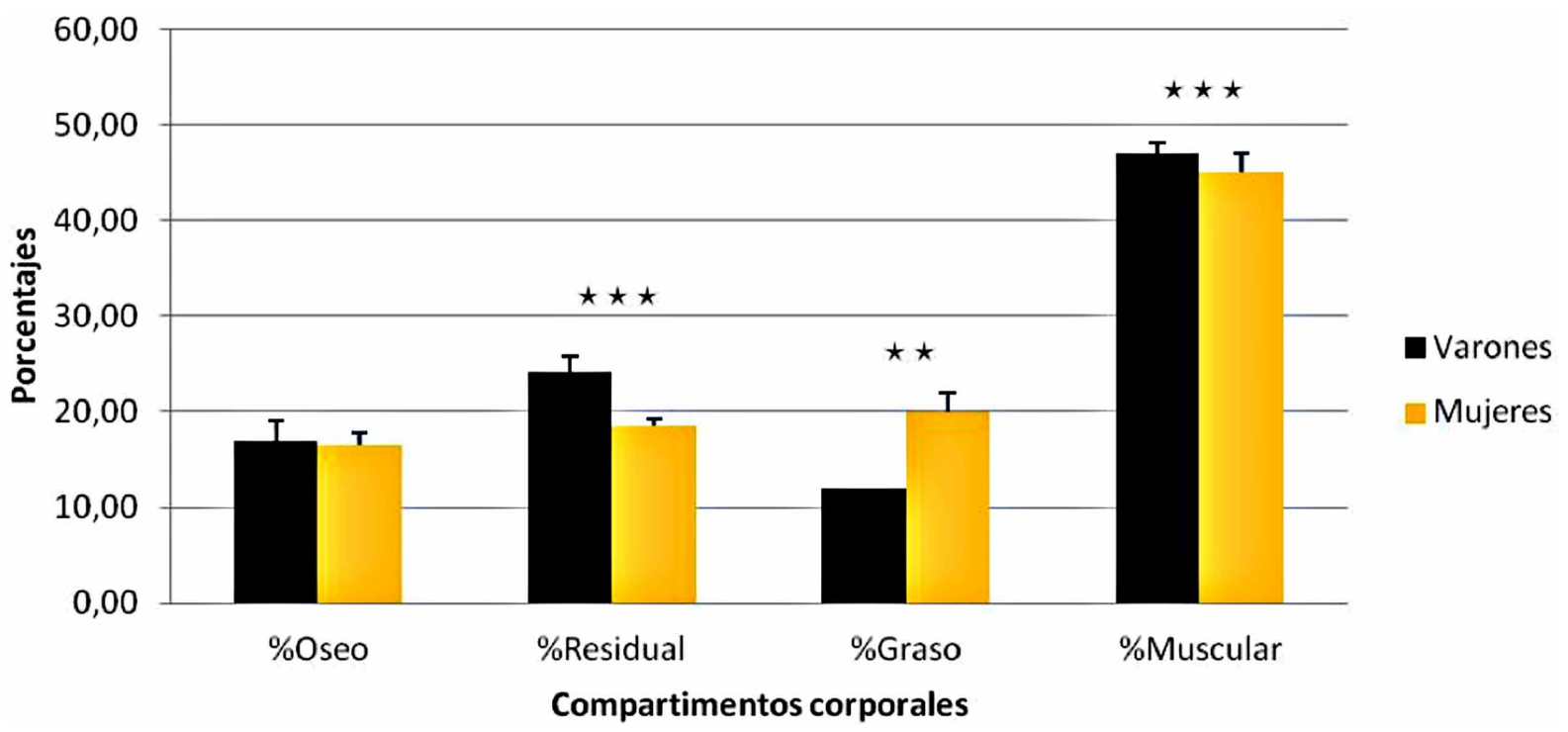

Fig. 2. Comparación de composición corporal de los jugadores de tenis de mesa según sexo. *p<0,05. **p<0,01. ***p<0,001.

Tabla IV. Comparación de composición corporal y somatotipo según sexo

\begin{tabular}{lccccc}
\hline & \multicolumn{2}{c}{ Varones $(\mathbf{n}=\mathbf{1 8})$} & \multicolumn{3}{c}{ Mujeres $(\mathbf{n = 1 3})$} \\
\hline Variable & Media (DE) & Rango & Media (DE) & Rango & Valor $\mathrm{p}$ \\
\% Peso graso & $12( \pm 2,7)$ & $7,8-16,8$ & $14,7( \pm 1,5)$ & $12,1-16,4$ & $<0,001$ \\
\% Peso muscular & $46,9( \pm 2,4)$ & $43,4-51,7$ & $45,7( \pm 2,3)$ & $42,6-50,3$ & $\leq 0,004$ \\
\% Peso óseo & $16,8( \pm 1,0)$ & $14,9-18,4$ & $16,5( \pm 1,2)$ & $14,5-18,2$ & $\mathrm{~ns}$ \\
\% Peso residual & $24,09( \pm 0,05)$ & $24-24,1$ & $18,5( \pm 0,5)$ & $17,8-19,5$ & $<0,001^{\ddagger}$ \\
Endomorfia & $2,5( \pm 0,8)$ & $1,6-4,2$ & $4,0( \pm 0,6)$ & $3,2-5$ & $<0,001^{\ddagger}$ \\
Mesomorfia & $4,6( \pm 1,2)$ & $3,3-6,7$ & $3,4( \pm 1,2)$ & $2,1-6,1$ & $<0,02$ \\
Ectomorfia & $2,6( \pm 1,0)$ & $0,9-4,2$ & $2,9( \pm 0,9)$ & $1,2-4,2$ & $\mathrm{~ns}$ \\
\hline
\end{tabular}

(Prueba t de Student; $\$$ Prueba U de Mann-Whitney; ns: no significativo). 


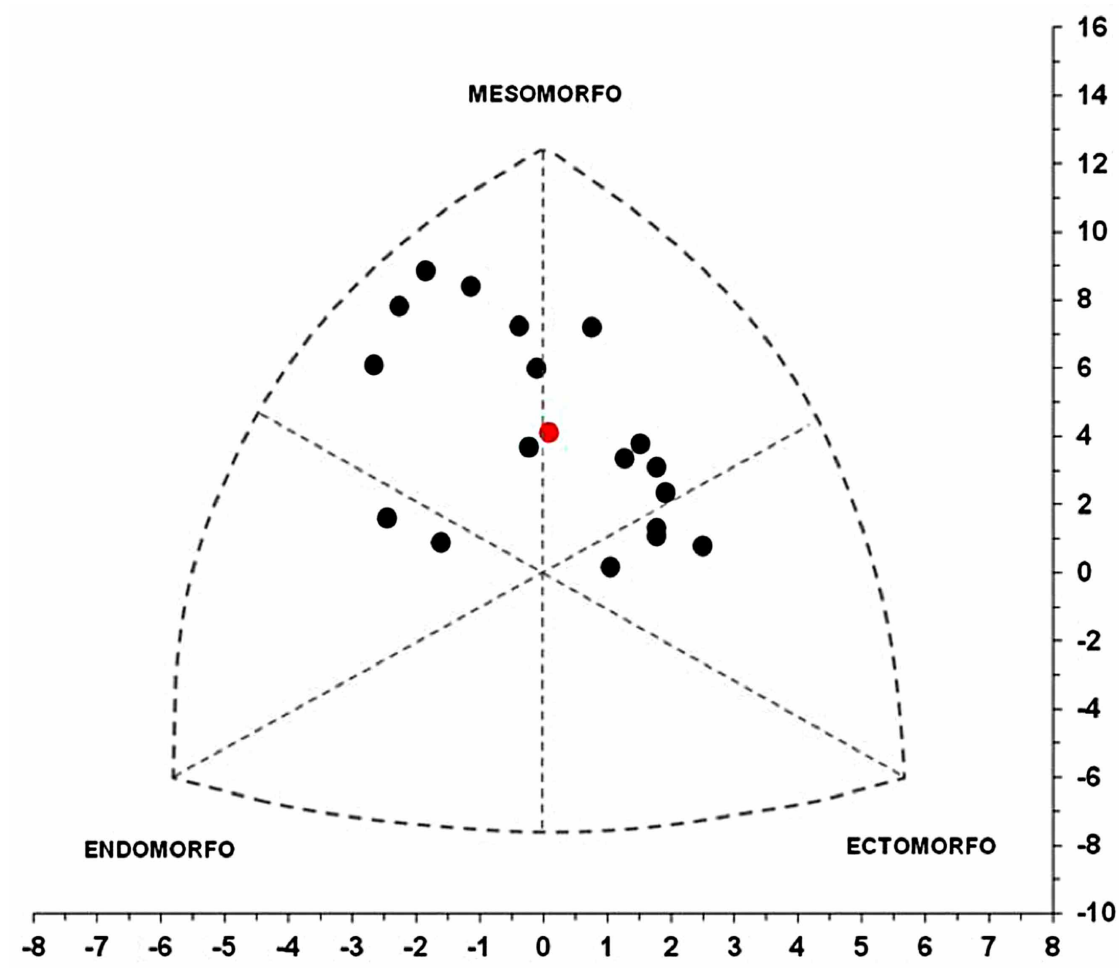

Fig. 3. Distribución del somatotipo masculino en la somatocarta $(\mathrm{n}=18)$. Somatotipo medio $=2,5-4,6-2,6$.

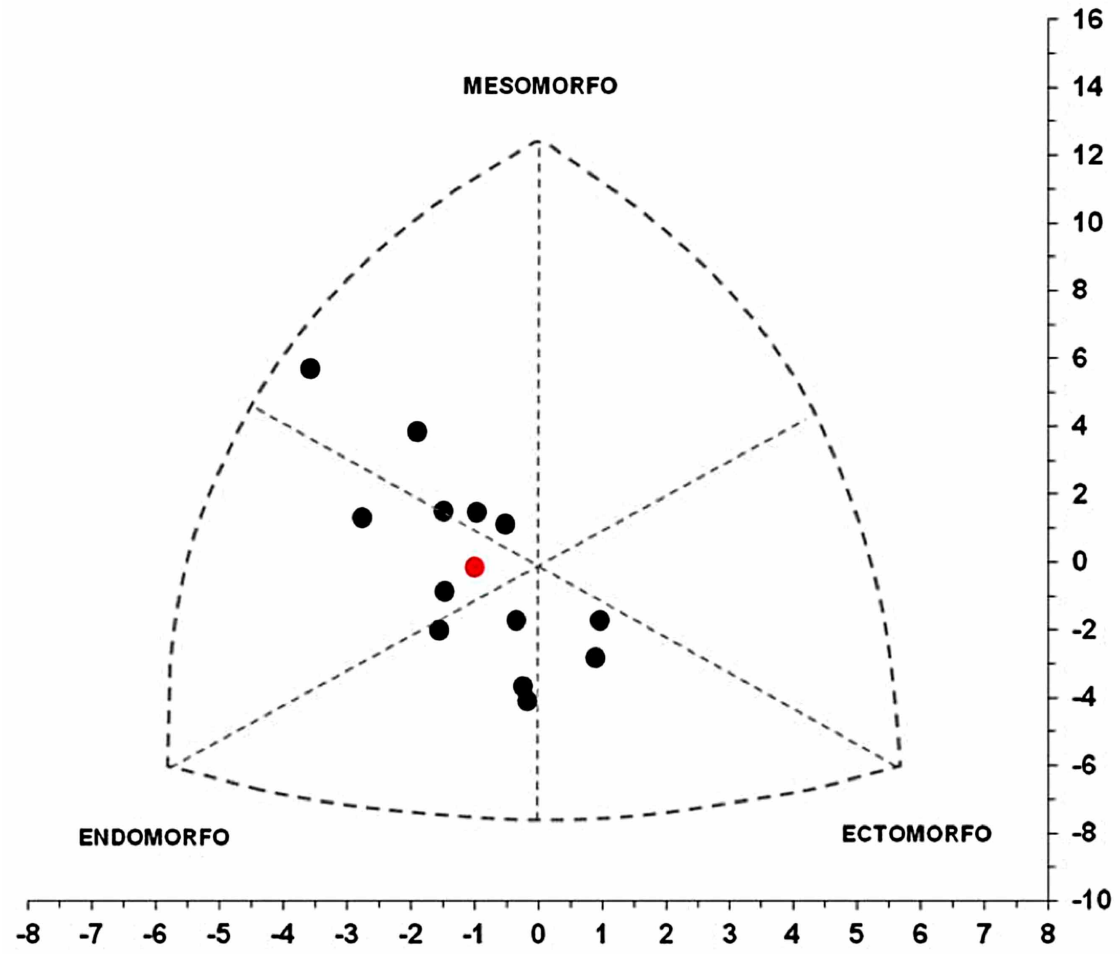

Fig. 4. Distribución del somatotipo femenino en la somatocarta $(n=13)$. Somatotipo medio $=4,0-3,4-2,9$. 


\section{DISCUSIÓN}

Diferentes estudios han analizado las características antropométricas, la composición corporal o el somatotipo en deportes de raqueta y pala como el bádminton (Centeno et al.; De Hoyo et al.; Faccini \& Dal Monte, 1996; Hughes et al., 1995; Ooi et al., 2009), el squash (Alvero et al.; Chin et al., 1995; Johansen \& Jensen, 1999; Mellor et al.; Todd \& Mahoney, 1995), o el tenis (Barbados-Tudor et al., 2011; Groppel \& Roetert, 1992; Hornery et al., 2007; Juzwiak et al.; Smekal et al., 2001; Sánchez-Muñoz et al.; Sanchís et $a l$.$) . Sin embargo, en el tenis de mesa en particular se en-$ cuentran escasas investigaciones que describan con exactitud los indicadores biotipológicos asociados al rendimiento que caracterizan a esta disciplina deportiva (Allen; Pradas et al., 2012b). Comparar los resultados obtenidos en esta investigación con otros datos similares que aborden en la literatura el tenis de mesa resulta complejo por diversas razones como la utilización de diferentes niveles de rendimiento de los deportistas analizados, la ausencia de estudios que analicen ambos sexos o diferencias en cuanto al número y edad de los participantes.

En el presente estudio los jugadores hombres mostraron valores superiores en las variables masa, talla e IMC. La altura media de los jugadores hombres $(177 \pm 0,06 \mathrm{~cm})$ fue superior al de las mujeres $(165 \pm 0,06 \mathrm{~cm})$. Los datos obtenidos para los hombres son similares a los hallados por Lentini et al. (2004) en 8 jugadores de elite argentinos de $24,9 \pm 6,9$ años de edad y por Allen en una muestra de 14 jugadores de alto nivel $(177,5 \pm 1,8)$. Sin embargo, estos valores son inferiores a los encontrados en otros deportes de raqueta como el bádminton $181 \pm 5,7 \mathrm{~cm}$ (Faccini \& Dal Monte), el tenis de alto nivel y el tenis a nivel profesional en donde se han encontrado estaturas de $1,83 \pm 6,9 \mathrm{~cm}$ y $1,84 \pm 7,15 \mathrm{~cm}$ respectivamente (Barbaros-Tudor et al.; Hornery et al.). En squash los jugadores de alto nivel analizados presentaron diferentes alturas $172,6 \pm 4,3 \mathrm{~cm}, 174,5 \pm 0,7$ cm y $182 \pm 7 \mathrm{~cm}$ (Chin et al.; Todd \& Mahoney; Johansen \& Jensen).

Los valores obtenidos en las mujeres son similares a los hallados por Allen en tenis de mesa $(164,5 \pm 2)$ y por Dias \& Ghosh (1995) y Hughes en bádminton en donde la altura de las deportistas se encontraba comprendida en un rango de 160-166,5 cm. Sin embargo, estos datos son inferiores a los encontrados en tenis $181 \pm 5,7 \mathrm{~cm}$ (Smekal et al.). Aunque la altura no es un componente esencial determinante para obtener el éxito deportivo en tenis de mesa, las palancas articulares largas con una buena coordinación intramuscular y poco peso corporal favorecen el desarrollo de una máxima fuerza explosiva, y por tanto la posibilidad de desarrollar desplazamientos segmentarios o globales muy rápidos, alcanzando elevadas velocidades que favorecen el rendimiento en este deporte (Pradas, 2002).

La masa corporal promedio de los jugadores de tenis de mesa fue de $71,9 \pm 9,1 \mathrm{~kg}$. Allen obtuvo valores ligeramente superiores en los jugadores analizados $(73,5 \pm 2,2)$. Datos similares fueron obtenidos por Lentini et al. en la

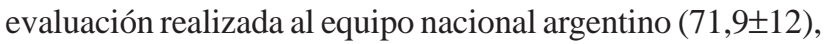
siendo ambos resultados muy superiores a los hallados en una muestra de jugadores españoles de tenis de mesa de elite de categoría junior $(57 \pm 10,5 \mathrm{~kg})$ (Martínez et al., 2009). Valores inferiores se obtuvieron en jugadores profesionales de tenis $(79,2 \pm 6,4 \mathrm{~kg})$, así como en jugadores de bádminton de elite de nacionalidad danesa y malaya en los que se encontraron pesos corporales que oscilaban entre los 73,3-76,9 kg y 73,2 $\pm 7,6$ kg respectivamente (Omosegaard, 1996; Ooi et al.). Estos resultados fueron superiores a los mostrados por Chin et al. en squash $(67,7 \pm 6,9 \mathrm{~kg})$ pero inferiores a los obtenidos en esta misma disciplina deportiva en investigaciones realizadas por Pyke et al. (1974) 76,8 $\pm 9 \mathrm{~kg}$ y Girard

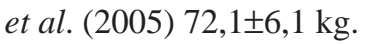

Las mujeres obtuvieron un valor medio en su masa corporal de 55,8 $\pm 5,1 \mathrm{~kg}$. Estos resultados coinciden con los hallados en jugadoras de alto nivel $(55,5 \pm 1,8 \mathrm{~kg})$ (Allen). Valores ligeramente inferiores fueron encontrados en una muestra de jugadores de tenis de mesa de elite de categoría junior 53,1 22,3 kg (Martínez et al.). Los resultados obtenidos en tenis de mesa se hallan por debajo de los alcanzados en jugadoras de elite de la selección danesa de bádminton (Omosegaard) encontrándose entre los 57-66,7 kg. En jugadoras de tenis austríacas de alto nivel se obtuvieron pesos más elevados 73,2 $\pm 6,8 \mathrm{~kg}$ (Smekal et al.). Un excesivo peso corporal en un jugador de tenis de mesa genera una clara desventaja frente al adversario produciéndose un efecto negativo sobre el rendimiento, fundamentalmente para realizar desplazamientos específicos. Masas corporales elevadas suponen una realización de esfuerzos musculares del tren inferior muy severos, por el grado de aceleración y desaceleración que requieren para posicionarse correctamente, golpear el móvil y regresar a la posición ideal en el terreno de juego, todo ello a una máxima velocidad de desplazamiento (Pradas et al. 2005).

Todos los pliegues cutáneos evaluados fueron inferiores en hombres. Las diferencias son más acusadas en los pliegues correspondientes a los miembros. Estos resultados concuerdan con los expresados por Malina \& Bouchard (1991) en donde indican que las diferencias sexuales son más acentuadas en los pliegues de los miembros que en los del tronco. El sumatorio de 6 pliegues se comporta de forma parecida siendo este resultado mayor para el sexo femeni- 
no. Las diferencias encontradas en cuanto a la masa, talla, IMC, perímetros y diámetros se relacionan directamente con el dimorfismo sexual existente entre hombres y mujeres (Lewis et al., 1986; Cox \& Calsbeek, 2010; Kirchengast, 2010)

El porcentaje de peso graso (PG) fue inferior en los hombres $(12 \pm 2,7 \%)$ respecto a las mujeres $(14,7 \pm 1,5 \%)$. Estos datos coinciden con investigaciones de índole similar como la realizada en tenis por Groppel \& Roetert (1992). El PG de los hombres $(12,09 \pm 1,07 \%)$ es similar al hallado en tenis de mesa por Allen (1991). En bádminton Majumdar et al. (1997) y Ooi et al. analizaron a jugadores pertenecientes al equipo nacional de la India $(12,1 \pm 3,4 \%)$ y de elite de Malasia $(12,5 \pm 4,8)$ obteniendo resultados equivalentes. En squash se han hallado valores de PG que van desde los

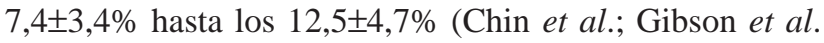
1999). En tenis los datos obtenidos respecto al PG en jugadores de alto nivel apuntan hacia porcentajes inferiores al 12\% (Barbaros-Tudor et al.).

El PG de las mujeres de tenis de mesa analizadas en este estudio es inferior al obtenido por Allen en donde se obtuvieron porcentajes del 18,55 $\pm 0,7 \%$. Investigaciones realizadas sobre jugadoras de categoría sénior de bádminton y de tenis mostraron valores de $23,6 \pm 3,3 \%$ y $18,1 \pm 2,3 \%$ respectivamente (Hughes; Pyke et al.). El PG corporal puede considerarse para el jugador de tenis de mesa como un factor que puede limitar su rendimiento, tal y como señalan para el tenis Mahoney \& Sharp (1995). La grasa actúa como peso inerte y genera resistencia a ser desplazada de forma reiterada en contra de la gravedad, como ocurre durante los movimientos rápidos y explosivos que se producen en el transcurso del juego en tenis de mesa (Pradas et al., 2007). Además, tal y como apunta Chin et al. un exceso de grasa corporal podría aumentar el gasto de energía que se requiere durante un partido provocando un impacto negativo sobre el rendimiento físico y técnico-táctico que se va acentuando a medida que transcurre la competición.

Las diferencias halladas en las variables talla, masa corporal y en los porcentajes de grasa corporal en un mismo deporte podrían explicarse como consecuencia de que las investigaciones revisadas comparan a sujetos de diferentes poblaciones (caucásicos, asiáticos, etc.) ya que el porcentaje de grasa corporal es diferente en función del grupo étnico (Deurenberg et al., 1998).

El somatotipo obtenido por los hombres fue de tipo mesomorfo balanceado (2,5-4,6-2,6). Lentini et al. obtuvieron en jugadores de tenis de mesa de alto rendimiento argentinos un somatotipo endo-mesomórfico $(3,4-4,5-2,5)$. A pesar de las diferencias encontradas en el componente endomórfico, en ambos estudios el componente mesomórfico es el predominante. Estos resultados concuerdan con los estudios realizados con jóvenes jugadores de alto nivel, siendo el aspecto mesomórfico algo más evidente principalmente en los hombres (Martínez et al.). Los jugadores analizados se distribuyen más por toda la somatocarta que las mujeres que se sitúan más en la zona central y en los sectores endomórficos de manera similar a otros estudios (Solanellas et al.). Las jugadoras en su somatotipo se aproximan más al de tipo central con un predominio endomorfo $(4,0-3,4-2,9)$.

Se han hallado diferencias significativas entre ambos sexos en la mayoría de las características antropométricas analizadas. El somatotipo obtenido por los hombres es mesomorfo balanceado $(2,5-4,6-2,6)$ mientras que el de las mujeres es de tipo central con una dominancia endomórfica (4,0-3,4-2,9). La mesomorfia es el componente predominante en los hombres y la endomorfia en las mujeres. Los resultados de la composición corporal por grupos mostraron un mayor porcentaje graso en mujeres respecto a hombres, y un porcentaje óseo y muscular ligeramente más elevado en el grupo de hombres con respecto al de mujeres.

La comparación de los somatotipos obtenidos con el de referencia (Esparza, 1993) mediante el cálculo de la distancia de dispersión del somatotipo (DDS) apunta a que no existen diferencias entre los somatotipos comparados (DDS $\geq 2$ ), si bien en las mujeres se encuentra muy próximo a alcanzar diferencias estadísticamente significativas $(1,94)$ sugiriendo por tanto que existen modificaciones respecto a su somatotipo de referencia. Los resultados obtenidos apuntan a un desarrollo músculo-esquelético en hombres frente a un ligero predominio de la cantidad relativa de grasa en mujeres.

Como consecuencia de las modificaciones producidas en el reglamento de juego durante la última década, la información aquí expuesta se presenta como un nuevo valor de referencia a considerar para el control del entrenamiento y del rendimiento en el tenis de mesa. Los componentes de los somatotipos de hombres y mujeres no se diferencian de forma significativa aunque la distancia en el DDS de las mujeres si apunta diferencias. Se hace necesario realizar nuevos estudios en los que se discrimine la muestra en función del estilo de juego empleado por cada jugador (ofensivo, mixto y defensivo).

\section{AGRADECIMIENTOS}

A la Real Federación Española de Tenis de Mesa y a todos los deportistas que participaron en el estudio. 
PRADAS, F. ; GONZÁLEZ-JURAdO, A.; MOLiNA, E. \& CASTEllaR, C. Anthropometric characteristics, body composition and somatotype of high level table tennis players Int. J. Morphol., 31(4):1355-1364, 2013.

SUMMARY: The aim of this study was to analyze the anthropometric profile, body composition and somatotype of international toplevel table tennis players belonging to the 150 best world players. A total of 31 players (18 males and 13 females) belonging to the absolute category were evaluated. All of them played regularly in the "Superdivision", the highest Spanish table tennis championship category as well as in other official international championships (ETTU e ITTF). Participants were divided into two groups according to their sex and a total of 16 anthropometric variables were assessed. Statistically significant differences were recorded in: body mass, height and BMI values ( $\mathrm{p}<0.01$ ); seven skinfolds $(p<0.05)$, two girths $(p<0.01)$ and in all breadths $(p<0.01)$. Significant differences were also found between the somatotype mesomorph and endo-mesomorph profiles $(\mathrm{p}<0.02)$. A balanced mesomorph somatotype was found for males $(2.5-4.6-2.6)$ while females recorded a mesomorph-endomorph somatotype (4.0-3.4-2.9). These results highlight a different biotype if compared with reference data with less endomorphy between males and an improvement of endomorphy and less mesomorphy between females. This study provides new biotypology data that represent a suitable reference for elite table tennis players.

KEY WORDS: Table tennis; Elite; Anthropometry; Body composition; Somatotype.

\section{REFERENCIAS BIBLIOGRÁFICAS}

Allen, G. D. Physiological characteristics of elite Australian table tennis athletes and their responses to high level competition. J. Hum. Mov. Stud., 20(3):133-47, 1991.

Alvero, J. R.; Barrera, J.; Mesa, A. \& Cabello, D. Correlations of physiological responses in squash players during competition. En Lees, A.; Cabello, D. \& Torres, G. (Eds.). Science and Racket Sports IV (64-69). London, Routledge, 2006.

Barbaros-Tudor, P.; Matkovic, B. \& Rupcic, T. Morphological characteristics and physiological profile of the Croatian male tennis players. Sport Sci., 4(2):23-7, 2011.

Biener, K. \& Oechslin, M. Sportmedizinisches Profil des Tischtennisspielers. Deutsche Zeitschrift für Sportmedizin, 30(12):406-10, 1979.

Carter, J. E. L. \& Heath, B. H. Somatotyping: Development and applications. Cambridge, Cambridge University Press, 1990.

Centeno, R. A.; Naranjo, J. \& Guerra, V. Estudio cineantropométrico del jugador de bádminton de élite juvenil. Arch. Med. Deporte, 16(70):115-9, 1999.

Chin, M. K.; Steininger, K.; So, R. C.; Clark, C. R. \& Wong, A. S. Physiological profiles and sport specific fitness of Asian elite squash players. Br. J. Sports Med., 29(3):158-64, 1995.

Cox, R. M. \& Calsbeek, R. Sex-specific selection and intraspecific variation in sexual size dimorphism. Evolution, 64(3):798-809, 2010.

Deurenberg, P.; Yap, M. \& van Staveren, W. A. Body mass index and percent body fat. A meta analysis among different ethnic groups. Int. J. Obes. Relat. Metab. Disord., 22(12):1164-71, 1998.

De Hoyo, M.; Sañudo, B.; París, F. \& de la Fuente, L. Estudio del biotipo y la composición corporal en jóvenes jugadores de bádminton. $M D$ Revista científica en Medicina del Deporte, 7:9-14, 2007.

De Rose, E. H. \& Guimaraes, A. C. A model for optimization of somatotype in young athletes. En: Ostin, M.; Buenen, G. \& Simons,
J. (Eds.). Kinanthropometry II. Baltimore, University Park Press, 1980.

Dias, R. \& Ghosh, A. K. Physiological evaluation of specific training in badminton. En: Reilly, T.; Hughes, M. \& Lees, A. (Eds.). Science and Racket Sports (38-43). London, E \& FN Spon, 1995.

Esparza, F. Manual de cineantropometría. Pamplona, FEMEDE, 1993.

Faccini, P. \& Dal Monte, A. Physiologic demands of badminton match play. Am. J. Sports Med., 24(6):564-6, 1996.

Faccini, P.; Faina, M.; Scarpellini, E. \& Dal Monte, A. Il costo energetico nel tennistavolo. Scuola dello sport, 17:38-42, 1989.

Faulkner, J. A. Physiology of swimming and diving. En: Falls, H. (Ed.), Exercise Physiology. Baltimore, Academic Press, 1968.

Gibson, A.; Lambert, M. I.; Hawley, J. A.; Broomhead, S. A. \& Noakes, T. D. Measurement of maximal oxygen uptake from two different laboratory protocols in runners and squash players. Med. Sci. Sports Exerc., 31(8):1226-9, 1999.

Girard, O.; Sciberras, P.; Habrard, M.; Hot, P.; Chevalier, R. \& Millet, G. P. Specific incremental test in elite squash players. Br. J. Sports Med., 39(12):921-6, 2005.

Groppel, J. L. \& Roetert, E. P. Applied physiology of tennis. Sports Med., 14(4):260-8, 1992.

Hornery, D. J.; Farrow, D.; Mujika, I. \& Young, W. An integrated physiological and performance profile of professional tennis. Br. J. Sports Med., 41(8):531-6, 2007.

Hughes, M. G. Physiological demands of training in elite badminton players. En: Reilly, T.; Hughes, M. \& Lees, A. (Eds.). Science and Racket Sports (38-43). London, E \& FN Spon, 1995.

Johansen, L. \& Jensen, K. Physical capacity of Danish elite squash players. Med. Sci. Sports Exerc., 31(5):S256, 1999.

Juzwiak, C. R.; Amancio, O. M.; Vitalle, M. S.; Pinheiro, M. M. \& Szejnfeld, V. L. Body composition and nutritional profile of male adolescent tennis players. J. Sports Sci., 26(11):1209-17, 2008. 
PRADAS, F. ; GONZÁLEZ-JURADO, A.; MOLINA, E. \& CASTELlAR, C. Características antropométricas, composición corporal y somatotipo de jugadores de tenis de mesa de alto nivel Int. J. Morphol., 31(4):1355-1364, 2013

Kirchengast, S. Gender Differences in Body Composition from Childhood to Old Age: An Evolutionary Point of View. J. Life Sci., 2(1):1-10, 2010.

Lentini, N. A.; Gris, G. M.; Cardey, M. L.; Aquilino, G. \& Dolce, P. A. Estudio somatotípico en deportistas de alto rendimiento de Argentina. Arch. Med. Deporte, 21(104):497-509, 2004.

Lewis, D. A.; Kamon, E. \& Hodgson, J. L. Physiological differences between genders. Implications for sports conditioning. Sports Med., 3(5):357-69, 1986.

Mahoney, C. A. \& Sharp, N. C. The physiological profile of elite junior squash players. En: Reilly, T.; Hughes, M. \& Lees, A. (Eds.). Science and Racket Sports (76-80). London, E \& FN Spon, 1995.

Majumdar, P.; Khanna, G. L.; Malik, V.; Sachdeva, S.; Arif, M. \& Mandal, M. Physiological analysis to quantify training load in badminton. Br. J. Sports Med., 31(4):342-5, 1997.

Malina, R. \& Bouchard, C. Growth maturation and physical activity. Champaign, Illinois, Human Kinetics, 1991.

Martínez, E.; Carrasco, L.; Alcaraz, P. E.; Pradas, F. \& Carrillo, J. P. Perfil antropométrico, somatotipo, composición corporal y dinamometría manual en jóvenes jugadores de alto nivel de tenis de mesa. Act. Fis. Dep. Cienc. Prof., 10:55-60, 2009.

Mellor, S.; Hughes, M.; Reilly, T. \& Robertson, K. Physiological profiles of squash players of different standards. En Reilly, T.; Hughes, M. \& Lees, A. (Eds). Science and Racket Sports. London, E \& FN Spon, 1995. pp.72-5.

Omosegaard, B. Physical Training for Badminton. Denmark, Malling Beck, 1996.

Ooi, C. H.; Tan, A.; Ahmad, A.; Kwong, K. W.; Sompong, R.; Ghazali, K. A.; Liew, S. L.; Chai, W. J. \& Thompson, M. W. Physiological characteristics of elite and sub-elite badminton players. J. Sports Sci., 27(14):1591-9, 2009.

Pradas, F. De la iniciación al perfeccionamiento en el juego de dobles. Un caso práctico en tenis de mesa. En: D. Cabello (Ed.), Fundamentos y enseñanza de los deportes de raqueta y pala. Granada, Facultad de Ciencias de la Actividad Física y del Deporte, Universidad de Granada, 2002

Pradas, F.; de Teresa, C. \& Vargas, M. C. Evaluation of the explosive strength and explosive elastic forces of the legs in high level table tennis players. Sport Sci. Res., 3:80-5, 2005.

Pradas, F.; Carrasco, L.; Martínez, E. \& Herrero, R. Perfil antropométrico, somatotipo y composición corporal de jóvenes jugadores de tenis de mesa. Rev. Int. Cienc. Deporte, 3(7):11-23, 2007.

Pradas, F.; Rapún, M.; Martínez, P.; Castellar, C.; Bataller, V. \& Carrasco, L. An analysis of jumping force manifestation profile in table tennis. Int. J. Table Tennis Sci., 7:19-23, 2012a.

Pradas, F.; Floría, P.; González-Jurado, J. A.; Carrasco, L. \& Bataller, V. Developement of an observational tool for single table tennis analysis. J. Sport Health. Res., 4(3):255-68, 2012 b.
Püschel, K. Leistungsphysiologische Aspekte des Tischtennisspiels. Deutsche Zeiftschrift für sportmedizin, 12:357-60, 1978.

Pyke, S.; Elliott, C. \& Pyke, E. Performance Testing of Tennis and Squash Players. Br. J. Sports Med., 8(2-3):80-6, 1974.

Ripoll, H. Comprendere ed agire. Scuolla dello Sport, 8(17):22-8, 1989.

Rocha, M. S. L. Peso ósseo do brasileiro de ambos os sexos de 17 a 25 anhos. Arq. Anat. Antrop., 1:445-51, 1975.

Ross, W. D. \& Marfell-Jones, M. J. Kinanthropometry. En: MacDougall, J. D.; Wenger, H. A. \& Green, H. J. (Eds.). Physiological Testing of Elite Athlete. London, Human Kinetics, 1991. pp.223-308.

Shepard, R. J. \& Astrand, P. O. La resistencia en el deporte. Barcelona, Paidotribo, 1996.

Smekal, G.; Von Duvillard, S. P.; Rihacek, C.; Pokan, R.; Hofmann, P.; Baron, R.; Tschan, H. \& Bachl, N. A physiological profile of tennis match play. Med. Sci. Sports Exerc., 33(6):999-1005, 2001.

Sánchez-Muñoz, C.; Sanz, D. \& Zabala, M. Anthropometric characteristics, body composition and somatotype of elite junior tennis players. Br. J. Sports Med., 41(11):793-9, 2007.

Sanchís, J.; Dorado, C. \& Calbet, J. A. Regional body composition in professional tennis players. En: Lees, A.; Maynard, I.; Hughes, M. \& Reilly, T. (Eds.). Science and Racket Sports II. London, E \& FN Spon, 1998. pp. 34-40.

Solanellas, F.; Tuda, M. \& Rodríguez, F. A. Valoración cineantropométrica de tenistas de diferentes categorías. Apunts, Educ. Fís. Deport., 4445:122-33, 1996.

Tang, H. P.; Mizoguchi, M. \& Toyoshima, S. Speed and spin characteristics of the $40 \mathrm{~mm}$ table tennis ball. Int. J. Table Tennis Sci., 5:278-284, 2001

Todd, M. K. \& Mahoney, C. A. Determination of pre-season physiological characteristics of elite male squash players. En: Reilly, T.; Hughes, M. \& Lees, A. (Eds.). Science and Racket Sports. London, E \& FN Spon, 1995. pp.81-6.

Torres-Luque, G.; Alacid, F.; Ferragut, C. \& Villaverde, C. Estudio cineantropométrico del jugador de tenis adolescente. CCD, 2(4):2732, 2006.

Würch, A. La femme et le sport. Med Sport Française, 4:441-5, 1974.

Dirección para Correspondencia:

Francisco Pradas de la Fuente

Facultad de Ciencias Humanas y de la Educación

Universidad de Zaragoza

Calle Valentín Carderera 4

CP. 22003, Huesca

ESPAÑA

Email: franprad@unizar.es

Recibido : 10-04-2013

Aceptado: 28-08-2013 